\title{
The value of mediator soft skills to modern commercial practice
}

\section{Greg Rooney}

\section{Abstract}

This paper explores the nature of change in modern economies due to their growing interconnectedness and implications this has for the way professions such as mediation and legal services carry out their business. It argues that the biggest upheaval has been the rise in the commercial value of trust over that of competitive and adversarial behaviours and that this requires, in turn, that dealing with conflict takes account of the inherent human complexity found in trusting relationships. It contrasts the role of mediators in dealing with complexity and ambiguity with that of other professions, notably in the legal sphere. It notes attempts within the legal profession to rebadge itself away from litigation to dispute resolution and to promote evaluative mediation and semi-determinative processes as the pre-eminent conflict resolution process. It argues that the traditional non-evaluative 'process' approach to mediation is far more in tune with the modern collaborative economy and concludes that the legal profession should seek to learn from it and seek to adopt the soft skills of the mediator.

\section{Introduction}

Mediation has an identity issue - but it's not its fault. Mediators - and the profession they practise called mediation - merely sit as innocent bystanders observing how the modern collaborative interconnected economy has challenged the identity and, in some cases, the very existence of the established professions and commercial and social institutions.

We now live in a time where a world of connectivity and fluidity has replaced the $20^{\text {th }}$ century Newtonian concepts that are linear, predictable and 
deterministic. This is a world driven by the rise of the World Wide Web in 1990, powered by the Google search engine in 1996 and the power of social networking, starting with Facebook in 2004.

The world dramatically changed again in 2007 when Napster introduced the first sharing platform, heralding the beginning of the collaborative sharing economy. Then came Airbnb and Uber, both in 2008. We now have transparent and open data networks that are available free-of-charge to anyone with an Internet connection.

The latest iteration in this change is the development of the 'Internet of Things' which is a network of physical devices, including vehicles, home appliances and other items embedded with electronics, software, sensors, actuators and connectivity, which enables these things to connect with each other and exchange data. As an example, Uber's computers share traffic data with Google Maps computers.

The complexity caused by this connectivity has upended the Newtonian concept that the world is ordered and measurable and that having knowledge of the past will allow a computation of the future. Many organisations and professions, including the legal profession, still rely on a fixed Newtonian view of the world and wonder why they are being disrupted to their detriment.

The biggest upheaval has been the rise in the commercial value of trust over that of competitive and adversarial behaviours. The sharing economy relies on the willingness of users to be trustworthy and to trust each other. The platforms themselves also must be trustworthy. The sharing economy is built on the human element which is inherently complex. It is therefore essential that any conflict be dealt with in a way that preserves those trusting relationships while allowing new learnings which are an essential springboard for innovation and evolutionary breakthroughs.

We therefore require a new way of thinking and operating that can work with this complexity. 


\section{Challenge of the new collaborative order}

This complexity of this new world order accurately highlights the lot of the practising mediator. Mediators around the world will go off to work tomorrow morning and engage with parties at a very human level in much the same way as they have been doing since the late 1980s. They will work with the uncertainties of the conflict they are mediating and hopefully come up with 'good enough' resolutions. They will continue to deal with complexity and ambiguity daily and use their soft skills to massage impasses and blockages. These 'soft' skills are now in high demand in the commercial world.

Meanwhile, the established professions and the commercial and social institutions are having the identity crisis. They are in a scramble to find meaning and understanding to try to fit in with this new reality.

No profession is more under threat from this new world order than the legal profession. Since the 1980s, it has moved from being a trusted profession based on the application of scale costs, which moderated the profession's financial selfinterest, to a commercial business model built on time costing to maximise dollar return through promoting (litigation finance) and extending disputes by means of an adversarial culture. The problem is not so much the high legal fees, although this is an issue, it is the pursuit of the adversarial approach to drive those extra fees. This keeps their clients stuck in the conflict zone far longer than is commercially necessary.

This has turned out to be a huge self-inflicted wound. It has left the profession exposed and unprepared for the arrival of the open sharing economy built on trust and maintained by the soft skills of managers and their advisors.

\section{Re-badging the legal profession}

In response to the challenge of trying to provide value to the new collaborative economy, the legal profession has sought to rebrand itself to try to recover commercial relevance. It has looked to the trusted mediation movement as its path to restoring that lost trust. The legal profession has sought to rebadge itself by dumping the designation "litigators" and replacing it with "dispute resolvers" (DR) now with lofty ideals: 
"Through a fidelity to the good of DR, lawyers not only contribute constructively to society but they can also achieve positive interpersonal and individual change for their clients. This positive impact has the potential to extend to healing, wholeness, harmony and optimal human functioning." (Boulle and Field, 2018)

It is ironic that the long-standing criticism of traditional mediation by the legal profession has been that it is too 'touchy-feely' and into "healing, wholeness, harmony and optimal human functioning". These appear to be the very things that it is now trying to champion through its trumpeting of DR.

The proponents of the DR push have gone much further than a simple rebranding exercise. They have chosen to question and diminish the intent and identity of the traditional mediation movement.

Firstly, Boulle and Field (2018) suggest that, despite mediation's versatility and diversity of applications, it is not clear how mediators will respond to the challenges ahead. Secondly, they suggest it follows that this uncertainty for the future somehow renders mediation vulnerable to being subverted, rejected and replaced or modified beyond recognition. They then conclude that because of this uncertainty for the future, we should not pine for or have nostalgic sentiment for mediation's (presumably lost or invalid) original intent and identity.

The real intent of the dispute resolution movement is revealed by the assertion that:

"It will be necessary to use research to ensure that if evaluative mediation

becomes the normative approach, as well it might, that quality-control and ethical frameworks exist to prevent rogue mediators making de facto determinations." (highlights inserted) (Boulle and Field, 2018)

The recent Global Pound conferences (International Mediation Institute, 2018) were essentially a public relations exercise to pursue this end. These series of meetings held in 24 countries engaged various commercial stakeholders with traditional legal service providers involved in commercial dispute resolution (formerly known as litigation). It noted the resistance to change from external lawyers, particularly to adopting collaboration practices and non-adjudicative 
processes like mediation and conciliation. I am not sure the commercial world is buying this 'dispute resolution' makeover from traditional legal providers particularly when it is still built on an adversarial solution-focused culture that is not in harmony with modern economic drivers.

I would argue that the traditional non-evaluative 'process' approach to mediation is far more in tune with the modern collaborative economy. It is an experiential approach which gives the parties the time and space to step back and allow patterns to emerge. The mediator can sense and respond to these patterns. This creates the potential for new opportunities to emerge out of the interaction that can lead to innovation and creativity. It can help repair disrupted trust which is the central foundation of the modern economy. It is mediating for the emergence of the new rather than providing an evaluation of the parties' respective positions in order to close the gap.

The core facilitative skills that mediators acquire through the practice of sensing and responding to the immediacy of the moment equip them with the exact soft skills that the commercial world needs to manage in this complex environment. This is reflected in the fact that most MBA courses run throughout Australia have now been redesigned to incorporate soft skills as a core component of their coursework. Further, the big four accounting firms have created legal departments based on a collaborative non-litigious approach to providing legal expertise.

\section{Sir Isaac Newton is dead}

There is much of the Newtonian thinking underpinning those promoting the dispute resolution (DR) product. This can be seen in Boulle and Field (2018), where they connect measurement with understanding, a classic Newtonian concept. For example, Boulle and Field propose that it is only by evaluation and measurement that the legitimacy and credibility of mediation can be assessed. This is based on the Newtonian concept that the world is ordered and that if enough research is done, and a full understanding of a situation is achieved, then the future can be predicted. 
The challenge to this Newtonian view of the world can be seen in the replication movement in which many of the significant social science experiments of the past are being repeated - with vastly different results from the original conclusions (Ball, 2018). This is because nothing is repeatable in a complex environment. It has thrown into doubt the validity of much of the so-called evidence-based research and observational case studies carried out in the social science field. This has become a significant problem for academia.

The Newtonian view of the world has been superseded by modern physics; particularly, the laws of thermodynamics and the emerging awareness of quantum physics. These offer far better explanations of what is happening and, more importantly, why the world has changed so much.

The laws of thermodynamics hold the best scientific explanation of the disruptive world we live in. Thermodynamics is a branch of physics which focuses on the study of systems and how they interrelate. The first law of thermodynamics is that nothing is created or destroyed; it simply changes form. The second law of thermodynamics asserts that this change is always in the direction of decay and that all natural processes lead to an overall increase in disorder. It is why human beings, and nature in general, cannot reverse the ageing process.

As this change occurs nothing is lost or destroyed. It is simply reconstituted in another form which then becomes the new paradigm, before it too starts to decay. Disruption is therefore a normal part of reality and this conflicts with the ordered Newtonian view of the world that existed pre-2007.

The emerging understanding of quantum physics also impacts on our understanding of the complex world we now inhabit. It is a branch of physics which is highly uncertain and interconnected and where change occurs depending on the position of the observer. It breaks down the Newtonian link between cause and effect.

The answer to what will happen in the future in a complex environment cannot be found through analytical thinking. Outcomes cannot be predicted because in a complex environment every element is interconnected and these constantly co-constrain each other. It evolves in a random way through constant 
modification and never in the same way twice. Therefore, we can only understand what is happening in retrospect. No two contexts are the same and this makes it impossible to forecast or predict what will happen. J oining the dots in advance is an illusion.

This multi-faceted reality is a significant challenge for academia and theorists.

\section{Conclusion}

So tomorrow morning the traditional 'process' (non-evaluative) mediators will again go off to work where they will try to remain totally present in the moment to observe the dynamics of the interactions between parties in dispute. They will probe first and then sense and respond to the reaction and they will try and suspend any attachment to their memories, desires and the need to understand what is happening and will try not to be deterred by blockages and impasses. They will allow their intuition to guide them through the session rather than letting the mechanical side of their brain be the master (McGilchrist, 2009).

These are the same soft skills that leaders and managers in the commercial world need to use to manage the flow of networks between people in the way that allows for the safe space for minority views, diverging opinions, conflict and internal disruption to emerge. To develop these skills requires a higher state of alertness and the ability to provide a real-time response to emerging patterns and behaviours. This is the best pathway to creating strategic surprises and opportunities. If managers do not allow this type of internal disruption to occur then their competitors will disrupt externally.

For the legal community in general and the 'dispute resolvers' in particular, the answer to restoring value for the legal product in the new economy is not to push aside or try to diminish the traditional mediation movement but to embrace it, and welcome it as the path to acquiring the necessary soft skills to constructively engage with the fluidity, ambiguity and complexity of the new age.

I therefore suggest that rumours of the death of mediation and the significant role of the traditional process mediator are greatly exaggerated. 


\section{References}

Boulle, L. and Field, R. (2018), Mediation in Australia, LexisNexis

International Mediation Institute (2018) Global Pound Conference Series - Global Data Trends and Regional Differences, Report at:

file:/ / / C:/ Users/ PC/ AppData/Local/ Packages/ Microsoft. MicrosoftEdge_8wekyb3d8 bbwe/ TempState/ Downloads/ Global-Data-Trends-and-Regional-

Differences\%20(3).pdf

Ball, P. High-profile J ournals Put to Reproducibility Test, Nature, Springer Nature, Aug 27, 2018

McGilchrist, I. (2009) The Master and His Emissary, Yale University Press.

First published as a three-part blog on the Australian Dispute Resolution Research Network in October/ November 2018 https://adrresearch.net/

Greg Rooney has been a mediator in private practice in Australia for 27 years and has since 1995 taught mediation and allied ADR (Alternative Dispute Resolution subjects in a number of universities and private institutions in Australia and internationally. Greg has over the last 14 years mediated over 200 face-to-face meetings between religious leaders and individual victims of sexual abuse within a number of Christian religious institutions in Australia as well as abuse within the Australian Defence Force and the South Australian Police Force. Greg, together with colleagues Margaret Ross and Barbara Wilson, has since 2012 run an annual Mediation Retreat in Tuscany, Italy. 